\title{
Stick based Non-local Means Filter for Speckle Reduction in Ultrasonic Images
}

\author{
Ting Liu ${ }^{1}$, Nai-zhang Feng ${ }^{1}$, Yi Shen ${ }^{1}$, Ming-jian Sun ${ }^{1, a}$ \\ ${ }^{1}$ Harbin Institute of Technology, China
}

\begin{abstract}
Accurate visualization and quantification of human structure is an important prerequisite for a number of clinical procedures. Specially, a current challenging issue in medical ultrasonic images is the problem of speckle reduction while keeping the structure and texture information. A stick based non-local means filter is proposed in this paper. An asymmetric stick filter kernel is firstly defined by decomposing the rectangle search window of non-local means (NLM) filter into a set of line segments with variable orientations. Then, the sticks which used to search for similar pixels are selected by a normalized variance function. Finally, the weighted sum of averages of the similar pixels searched along each selected stick is used to produce the filtered image. With the introduction of the asymmetric stick, it is possible to implement the NLM filter in an oriented diffusion way. Experiments of synthetic and real clinical ultrasound images show that the stick based NLM filter performs effectively in suppressing speckle while pre-serving resolvable structures and even enhancing linear features such as the edges.
\end{abstract}

\section{Introduction}

Ultrasound imaging system is among the most widely used medical imaging modality. However, due to constructive and destructive coherent interferences of backscattered echoes from the scatters that are typically much smaller than the spatial resolution of ultrasound systems $^{[1]}$, a granular pattern is formed, namely speckle. It is considered as an undesirable property for it significantly degrades the images quality, and hence reduces the ability to discriminate fine details.

Such facts motivate large amount of researches ${ }^{[2-5]}$ dedicated to suppress the speckle. Besides the compounding technique, special focus has been given to post-filtering methods. No hardware modification is required and the existing images from general ultrasonic scanners can be directly applied. Adaptive spatial filter ${ }^{[6-}$ ${ }^{10]}$ is a kind of filtering technique for ultrasonic speckle reduction which utilizes the local statistics of speckle to effectively suppress speckle.

As a challenging adaptive spatial smoothing filter, NLM filter has been introduced by Buades et al. ${ }^{[1]}$ for 2D images with additional Gaussian noise. It is based on the idea that any natural image has redundancy, and that any pixel of the image has similar pixels that are not necessarily located in its spatial neighborhood. In this approach, the weight involving pixels in the average, does not depend on their spatial proximity to the current pixel but is based on the intensity similarity of their neighborhoods with the neighborhood of the pixel under study. The NLM has been applied to ultrasonic images by several groups ${ }^{[12-14]}$. Results from these methods are encouraging, but the computed efficiency and high sensitivity of the empirically selected parameters still remain problems. What's more, for the specificity of the speckle, the edge and detail information will be blurred.

Motivated by stick technique for line boundary detection $^{[15]}$, a stick based NLM filter has been proposed in which a new search window is defined from a set of asymmetric sticks, such that the new filter search for the most relevant pixels in an oriented diffusion way. The results are presented in a comparative way with several the state-of-the-art speckle reduction filters. In Section 2, the NLM filter is briefly reviewed. The stick based NLM filter is described with details in Section 3. In Section 4, experiments on both synthetic and clinical ultrasonic images are presented. Conclusion is drawn in section 5.

\section{NLM Filter}

For NLM filter, the restored intensity $N L(u)\left(x_{i}\right)$ of pixel $x_{i}$ is the weighted average of all the intensities in the image $u$, which is defined as

$$
N L(u)\left(x_{i}\right)=\sum_{x_{j} \in \Omega^{D}} w\left(x_{i}, x_{j}\right) u\left(x_{j}\right)
$$

where $D$ is the dimension $u ; u\left(x_{j}\right)$ is the pixel intensity and $w\left(x_{i}, x_{j}\right)$ is the weight assigned to $u\left(x_{j}\right)$ in the restoration of $x_{i}$. More precisely, the weight is

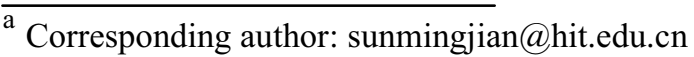


determined by the similarity of the local neighbourhood $N_{i}$ and $N_{j}$ of the pixels $x_{i}$ and $x_{j}$ which is computed by the Gauss-Weight Euclidean distance $\|\cdot\|_{2, a}^{2}$.

For computational efficiency, a search window $S_{i}$ is used to limit the number of pixels. Hence, the weight of each pixel $x_{j}$ in $S_{i}$ is defined as

$$
w\left(x_{i}, x_{j}\right)=\frac{1}{Z_{i}} e^{-\frac{\left\|u\left(N_{i}\right)-u\left(N_{j}\right)\right\|_{2, a}^{2}}{h^{2}}}
$$

where $Z_{i}$ is normalized constant, $h$ acts as a smoothing parameter controlling the degree of the filtering.

In [11], Buades et al. has shown that, for 2D natural images with additional noise, the NLM filter outperforms many other state-of-the-art denoising methods. Nevertheless, the NLM used for despeckling could not achieve as good performance as it does in the natural images. The edge is blurred while the image has patch like structure. So a stick based NLM filter is proposed to oriented implement the NLM to efficiency reduce the speckle while preserving the edge and local structure.

\section{The Stick based NLM Filter}

Originating from the work of Czerwinski et al., a selective smoothing along the line segments radiating outward from the investigated pixel is highlighted. Here, the idea is formulated in a different viewpoint, i.e. the orient ability of stick model and the local scale control of nonlinear diffusion are naturally integrated to NLM filter to develop an oriented adaptive speckle reduction filter.

\subsection{Asymmetric stick}

The stick method proposed by Czerwinski et al. is used for line boundary enhancement in ultrasonic images. The sticks are in essence line segments passing through the processed pixel, whose length is short enough to locally approximate edges, but long enough that the speckle along them is uncorrelated and thus their projections average the noise out. This technique can clearly enhance bright edges, but its noise-eliminating ability is restricted since it keeps the non-edge pixels unchanged in homogeneous regions.

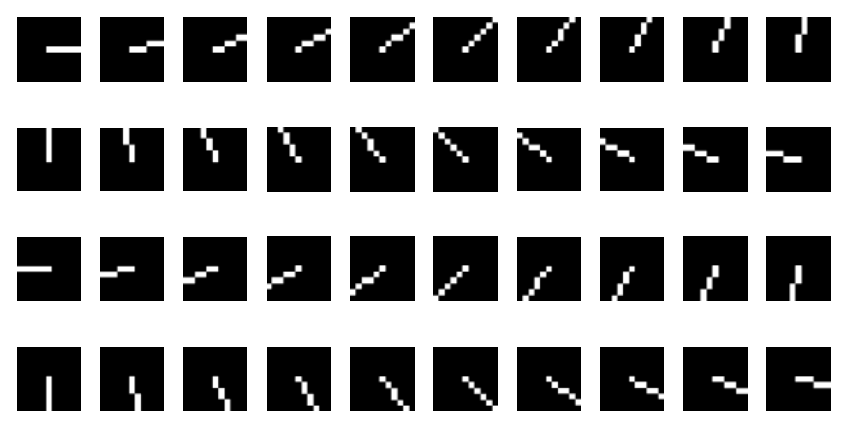

Fig. 1 Asymmetric sticks with 6 pixels length

Here, the idea of stick technique is merged into the NLM filter by dividing the search window into rays with varying angular orientation. The asymmetric stick is used instead of the original symmetrical sticks to encourage better intraregional smoothing. Fig. 1 shows a set of asymmetric sticks of 6-pixel length.

\subsection{The Stick based NLM method}

With asymmetric sticks, a function of the local variance along the sticks $g\left(V_{i}\right)$ is utilized to select sticks to form a new search window $S_{i s}$. Different kinds of $g(\square)$ can be adopted to do the selection. For example, we could simply take

$$
g\left(V_{i}\right)= \begin{cases}\frac{k}{V_{i}} & V_{i} \leq T \\ 0 & \text { otherwise }\end{cases}
$$

where $V_{i}$ is the local variance along the $i$ th stick, $T$ is the threshold. Take an individual search window into account, it can be intuitively explained that our model encourages smoothing along the sticks with relatively low variance. This idea is so similar with the diffusion function used in nonlinear diffusion filter. Hence we choose the nonlinear diffusion conductance function instead

$$
\begin{gathered}
g\left(V_{i}\right)=e^{-V_{i} / \sigma_{1}^{2}} \\
g\left(V_{i}\right)= \begin{cases}\frac{1}{2}\left[1-V_{i} / \sigma_{2}^{2}\right]^{2} & V_{i} \leq \sigma_{2}^{2} \\
0 & \text { otherwise }\end{cases}
\end{gathered}
$$

The threshold $\sigma_{1}^{2}$ and $\sigma_{2}^{2}$ could be set manually or estimated from the speckle and structure regions in ultrasonic images. The only discrepancy is that the original square of gradient is replaced by the variance along the sticks. Then the new search window is formed with selected sticks with different coefficients.

$$
S_{i s}=\sum_{i=1}^{4 N-4} g\left(V_{i}\right) * \text { stick }\{i\}
$$

where $N$ is the search window size and sticks $\{i\}$ is the $i$-th stick temple.

With the new search window, a stick based NLM filter can be developed, i.e.

$$
\begin{aligned}
& N L(u)\left(x_{i}\right)=\sum_{x_{j} \in S_{i s}} w\left(x_{i}, x_{j}\right) u\left(x_{j}\right) \\
& w\left(x_{i}, x_{j}\right)=\frac{1}{Z_{i}} g_{i j} e^{-\frac{\left\|u\left(N_{i}\right)-u\left(N_{j}\right)\right\|_{2, a}^{2}}{h^{2}}}
\end{aligned}
$$

where $g_{i j}$ is the coefficient in the searching window. Obviously, the stick based NLM is still a special adaptive filter. Specially, in homogeneous regions (labeled as A in Fig. 2), the variance of all the sticks are approximately equal and all the sticks are selected. It leads to approximately isotropic smoothing so that the speckle in this region will be maximum suppressed. What's more, due to the asymmetric sticks decomposition is partially overlapped, which corresponds to an implicit assignment of distance relative weights, our method will actually work like a Gaussian smoothing filter with larger weights near the center. Meanwhile, in heterogeneous regions as the B-E labels in Fig. 2, the information is expressed as 
many different structures such as the tube shapes, the line boundaries and the brunches. Our filter encourages smoothing along the sticks falling entirely inside the structures, and penalizes those across edges on the assumption that the former take comparatively low variance. Hence, the speckle could be effectively reduced while the edge and detail information could be preserved or even enhanced. What's more, the continuality of the edge could be improved.
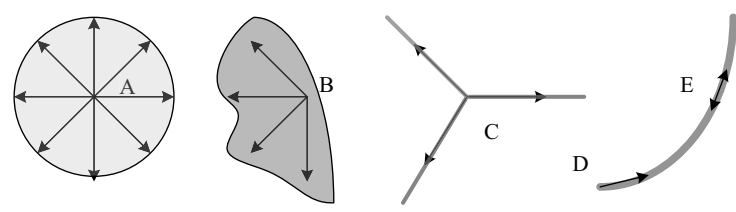

Fig. 2 The principal of the stick based NLM filter

\section{Experimental Results}

The performance of the stick based NLM filter is investigated with both synthetic and clinical images. Firstly, simulation studies are conducted for quantitative performance analysis. Then the result on a real ultrasound image demonstrates the effectiveness of the proposed method in real application.

\subsection{Synthetic Image}
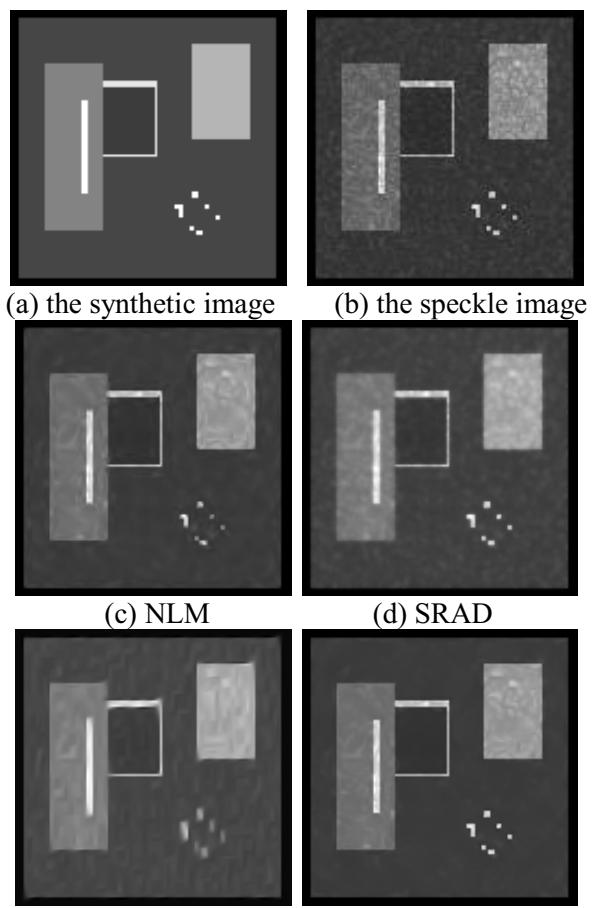

(d) SRAD

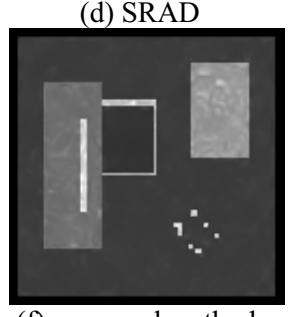

(e) NCD

(f) proposed method

Fig. 3 Results obtained with different filters applied to the synthetic image corrupted with signal-dependent speckle noise.

The speckle in ultrasonic images is different with addition and multiplicative noise. Several literatures have used the multiplication model to simulate it, but the model is not so appropriate. Here we adopt the method of
Ref.16. The synthetic image and that with speckles are shown in Fig. 3(a-b). The Peak signal-to-noise Ratio (SNR), the Mean Squared Error (MSE), the Mean Absolute Error (MAE) and the FOM are used to quantitatively evaluate the performance of the speckle reduction and the preservation of the edge.

As described, a set of asymmetric sticks are introduced to make NLM implicated in an oriented diffusion way. The proposed method is compared with NLM, SRAD and NCD, which are the most related stateof-the-art methods for speckle reduction in ultrasonic images. Table 1 gives the quantitative results and the despeckle images corresponding to speckle with $\sigma=0.4$ are presented in Fig. 3. Visually, the stick based NLM filter could effectively remove the speckle while preserving meaningful edges and the small details.

Table 1 the Comparing De-SPeCKle Result TABle of OBJeCtive EVALUATION

\begin{tabular}{ccccc}
\hline \hline Method & PSNR & MSE & MAE & FOM \\
Noisy image & 29.9111 & 66.3694 & 5.5744 & 0.8693 \\
NLM & 31.5915 & 45.0741 & 3.2600 & 0.8945 \\
SRAD & 30.7764 & 54.3801 & 4.2537 & 0.9403 \\
NCD & 31.1069 & 50.3949 & 3.8312 & 0.9298 \\
Our method & 35.4221 & 18.6582 & 2.4048 & 0.9482 \\
\hline \hline
\end{tabular}

\subsection{Clinical ultrasonic image}
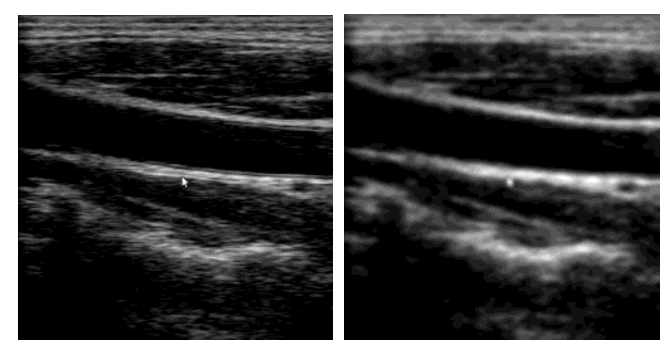

(a) Original ultrasound imag

(b) proposed method
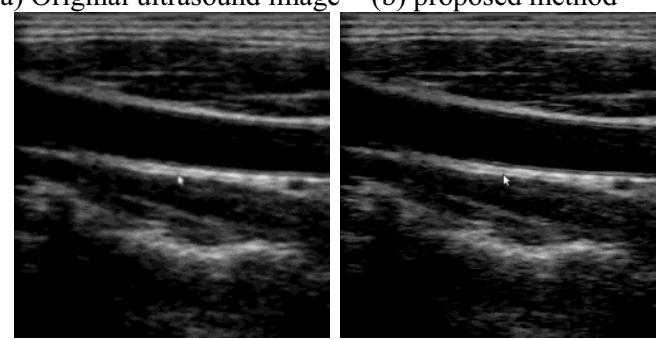

(c) image filtered by NLM (d) image filtered by SRAD

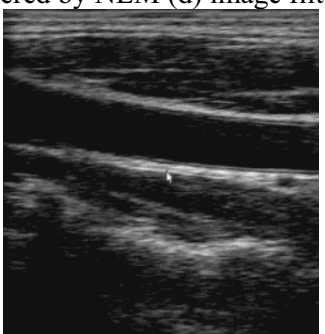

(e) image filtered by NCD

Fig. 4 Results obtained with different filters applied to the real clinical ultrasound image.

The original ultrasound image used is shown in Fig. 4 (a). In the proposed method, variation function $g_{3}(\cdot)$ with 
$\sigma_{2}^{2}=80$ is used. For the stick length, it should be longer than the speckle correlation length, but no longer than the length over which the edges are expected to be roughly straight. Here, the stick length is 9. Fig. 4(b) shows the result of our method.

For comparison, three filtering techniques were applied to the same image as shown in Fig.4(c)-(e). The same parameter with our method is set for NLM filter. For the SRAD and the NCD, the same parameters as [12] and [13] were used.

From Fig.4, it is obviously that our method could effectively suppress speckle while better preserving the edges. Specially, for NLM filter, the speckle cannot been effectively suppressed and the edge is discontinuity. For SRAD method, the edge is well preserved but the boundaries of bright regions are broadened. Although NCD filter enhances the coherence of organ surfaces, it causes blurring of small structures, and even enhances the speckle in homogeneous area.

\section{Conclusions}

A new speckle reduction method is presented for ultrasonic images. Unlike the previous techniques that depend on the identification of generally shaped image regions with contrasting statistical behavior, our method directly divided the search window of NLM into a set of asymmetric sticks. This naturally achieved an oriented diffusion implementation of NLM. Both synthetic and clinical ultrasound images, the new method outperformed the nonlinear diffusion models and NLM filter in terms of smoothing uniform regions and preserving feature details. In our filtered image, the edges become sharp, which will largely benefit the subsequent processing such as edge detection and image segmentation.

\section{Acknowledgements}

This research is supported by the National Natural Science Foundation of China (Grant No. 61201307, 61371045 and 61308116), the Fundamental Research Funds for the Central Universities (Grant No. HIT. NSRIF. 2013132), Science and Technology Development Plan Project of Shandong Province (Grant No.2015GGX103016) and China Postdoctoral Science Foundation (Grant No.2015M571413).

\section{References}

1. Burckhardt C B, IEEE Transaction on Sonics and Ultrasonics. 15 (1978)

2. Rahman M M, PK M K, Aziz A, et al, International journal of Covergence Computing. 1 (2013)

3. Mahmoud A A, Rabaie S E L, Taha T E, et al, Graphics and Signal Processing(IJIGSP). 5 (2013)

4. Esakkirajan S, Muhammed R, Subramanian G, Ultrasound in Medicine \& Biology. (2013)

5. T. Loupas, W. McDicken, and P. Allan, IEEE Trans. Circuits Syst. 36 (1989)
6. Lee, Jong-Sen, IEEE Transactions on Pattern Analysis and Machine Intelligence, PAMI-2 (1980)

7. Lopes, A., R. Touzi, and E. Nezry, IEEE Transactions on Geoscience and Remote Sensing. 28. 6 (1990)

8. Shi Z, Fung K B, Surface and Atmospheric Remote Sensing: Technologies, Data Analysis and Interpretation., International. IEEE. 4 (1994)

9. C. Tomasi and R. Manduchi, in ICCV '98: Proc. 6th Int. Conf. Computer Vision, Washington, DC. (1998)

10. Perona P, Malik J, Pattern Analysis and Machine Intelligence, IEEE Transactions on. 12 (1990)

11. Coupé P, Hellier P, Kervrann C, et al, Image Processing, IEEE Transactions on. 18. 10 (2008)

12. Coupé P, Yger P, Prima S, et al, Medical Imaging, IEEE Transactions on. 27. 4 (2008)

13. M. Mahmoudi and G. Sapiro, IEEE Signal Process. Lett. 12. 12 (2005)

14. Czerwinski, R.N., Jones, D.L., O Brien, W.D. IEEE Trans. Image Processing. 7 (1998)

15. A. Pizurica et al. IEEE Trans. Med. Imag. 22. 3 (2003)

16. J. A. Jensen, Med. Biol. Eng. Comput. 34 (1996)

17. Tay P C, Acton S T, Hossack J A, Biomedical Imaging: Nano to Macro, 2006. 3rd IEEE International Symposium on. IEEE (2006)

18. Tay P C, Garson C D, Acton S T, et al, Image Processing, IEEE Transactions on, 19. 7 (2010) 